\title{
Saturn's Rings,
}

the Yarkovsky Effects, and the Ring of Fire

by

David Parry Rubincam

Geodynamics Branch, Code 921

Laboratory for Terrestrial Physics

NASA Goddard Space Flight Center

Building 33, Room G308

Greenbelt, MD 20771

voice: $301-614-6464$

fax: 301-614-6522

email: David.P.Rubincam@nasa.gov

\section{June 2004}

One Sentence Summary:

The dimensions and the age of Saturn's main rings may be controlled by Yarkovsky forces, which also cause the particles in the $C$ ring to fall on the planet, where the luminous fireballs create a Ring of Fire around Saturn's equator. 


\begin{abstract}
The dimensions of Saturn's A and B rings may be determined by the seasonal Yarkovsky effect and the Yarkovsky-Schach effect; the two effects confine the rings between $\sim 1.68$ and $\sim 2.23$ Saturn radii, in reasonable agreement with the observed values of 1.525 and 2.267 . The $C$ ring may be sparsely populated because its particles are transients on their way to Saturn; the infall may create a luminous "Ring of Fire" around Saturn's equator. The ring system may be young: in the past heat flow from Saturn's interior much above its present value would not permit rings to exist.
\end{abstract}


What determines the dimensions of Saturn's ring system? Are the rings young or old? Below it will be shown that the interplay of two Yarkovsky forces predict that the outer edge of the A ring and the inner edge of the $C$ ring should be at certain distances from Saturn, and that these agree well with observation. The Yarkovsky forces also imply that the rings are relatively young.

The interplay is between the seasonal Yarkovsky effect, which makes the orbits of ring particles decay, and the Yarkovsky-Schach effect which, for random spin axis orientations, always makes the orbits expand. The seasonal and diurnal Yarkovsky effects have been extensively treated elsewhere $(1-4)$; a discussion of the Yarkovsky-Schach effect follows.

The Yarkovsky-Schach effect is about how solar thermal forces on the ring particles behave when the particles enter and exit Saturn's shadow $(5,0)$. The basic idea is qualitatively illustrated in Figure 1. The particle absorbs sunlight and then reemits it in the infrared. The infrared photons carry away more momentum from the hotter hemisphere than the colder one. The magnitude of the forces is indicated by the size of the thick arrows. When the particle dives into the shadow, the solar beating turns off, but the force takes some time to die away, due to the thermal inertia, and the particle continues to get a kick which increases the semimajor axis. As the particle emerges from the shadow, the force now acts against the motion so as to decrease the semimajor axis; but because the particle has cooled off, it takes some time for the particle to warm up and the force to turn back on. The net effect is a positive along-track acceleration which increases the particle's distance from Saturn. There are geometries for which the semimajor axis decreases; but for random spin axis orientations, the along-track forces do not average out to zero, resulting in a secular increase in the semimajor axis: the particle moves away from Saturn, increasing its angular momentum. A quantitative treatment of the Yarkovsky-Schach effect follows. 


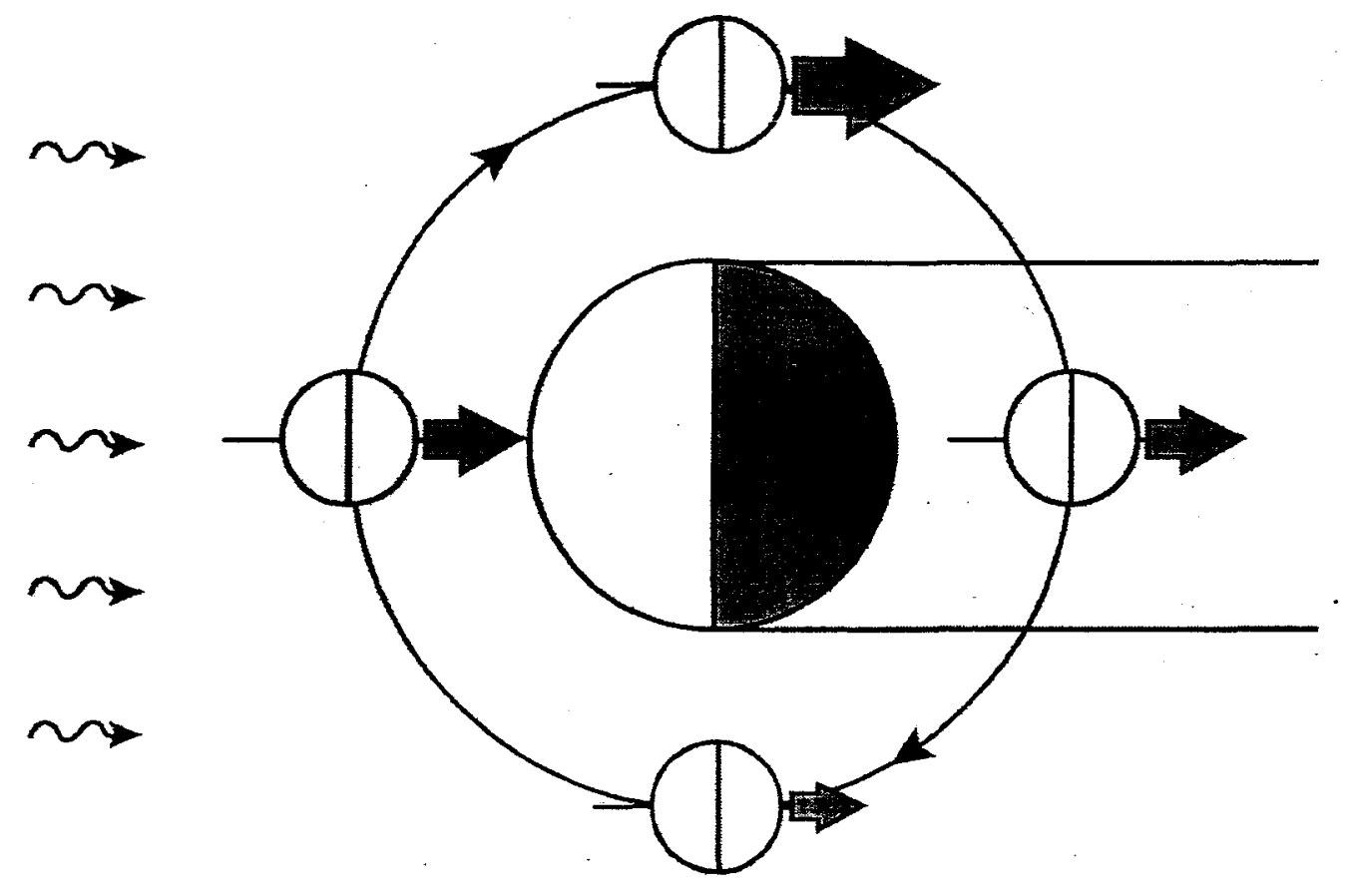

Figure 1. Schematic of the Yarkovsky-Schach effect. A ring particle orbits around Saturn; the view is looking up at Saturn's south pole. The particle's spin axis lies in the orbital plane and points to the Sun. The line across the particle is its equator. Sunlight comes in from the left and heats the sunward hemisphere, causing a thermal force (thick arrows). The size of the arrows indicates the magnitude of the force. The forces do not balance around the orbit because of the shadow and thermal inertia. The imbalance causes the orbit to expand secularly. There are also spin axis positions which cause the orbit to decay. However, averaging over all possible spin axis orientations causes the orbit to expand, as shown in the text.

The amount of sunlight falling on the particle will be given by $b(\phi) F_{S u n}$, where $F_{S u n}=$ $1371 \mathrm{~W} \mathrm{~m}^{-2} \times(1 \mathrm{AU} / 9.58 \mathrm{AU})^{2}=14.94 \mathrm{~W} \mathrm{~m}^{-2}$ is the solar insolation at Saturn's distance $(9.58 \mathrm{AU})$ from the Sun, and $b(\phi)$ is the "boxcar" function, which is equal to 1 when the particle is in full sunlight and 0 when it is in Saturn's shadow. Also, $\phi=n t$ is the angle in the equatorial plane from the $x$-axis (see Figure 2), where $t$ is time and $n=\left(G M_{s}\left(a^{3}\right)^{1 / 2}\right.$ is the mean motion, with $M_{S}=5.688 \times 10^{26} \mathrm{~kg}$ being the mass of Saturn and $G=6.67 \times 10^{-11} \mathrm{~m}^{3}$ $\mathrm{kg}^{-1} \mathrm{~s}^{-2}$ being the universal constant of gravitation. Simple Fourier analysis shows $b(\phi)$ to be 


$$
b(\phi)=b_{0}+\sum_{j=1}^{\infty} b_{j} \cos (j \phi)
$$

where $b_{0}=1-\Lambda$ and $b_{j}=2 \sin [j(1-\Lambda) \pi] /(j \pi)$ for all other terms, with $\Lambda$ being the fraction of the orbit which is shadowed. The parameter $\Lambda$ ranges from 0 (outside the shadow) to $1 / 2$ (hugging the planet).

Let $\hat{\mathbf{x}}, \hat{\mathbf{y}}$, and $\hat{\mathbf{z}}$ be the unit vectors along the respective axes in the reference frame shown in Figure 2. The Sun is stationed in the $x-z$ plane, so that the unit vector $\hat{\mathbf{r}}_{s}$ pointing from Saturn to the Sun is given by

$$
\hat{\mathbf{r}}_{S}=\sin \theta_{S} \hat{\mathbf{x}}+\cos \theta_{S} \hat{\mathbf{z}}
$$

where $\theta_{S}$ is the Sun's colatitude. Likewise, let $\hat{\mathbf{s}}$ be the unit vector in the direction of the particle's spin axis, so that

$$
\hat{\mathbf{s}}=\left(\sin \theta_{p} \cos \lambda_{p}\right) \hat{\mathbf{x}}+\left(\sin \theta_{p} \sin \lambda_{p}\right) \hat{\mathbf{y}}+\left(\cos \theta_{p}\right) \hat{\mathbf{z}}
$$

where $\theta_{p}$ is the axis' colatitude and $\lambda_{p}$ its east longitude. $\hat{\mathbf{r}}_{\mathrm{S}}$ and $\hat{\mathbf{s}}$ change slowly enough so that $\hat{\mathbf{r}}_{\mathbf{s}} \bullet \hat{\mathbf{s}}$ can be considered constant over one revolution of the particle about Saturn. 


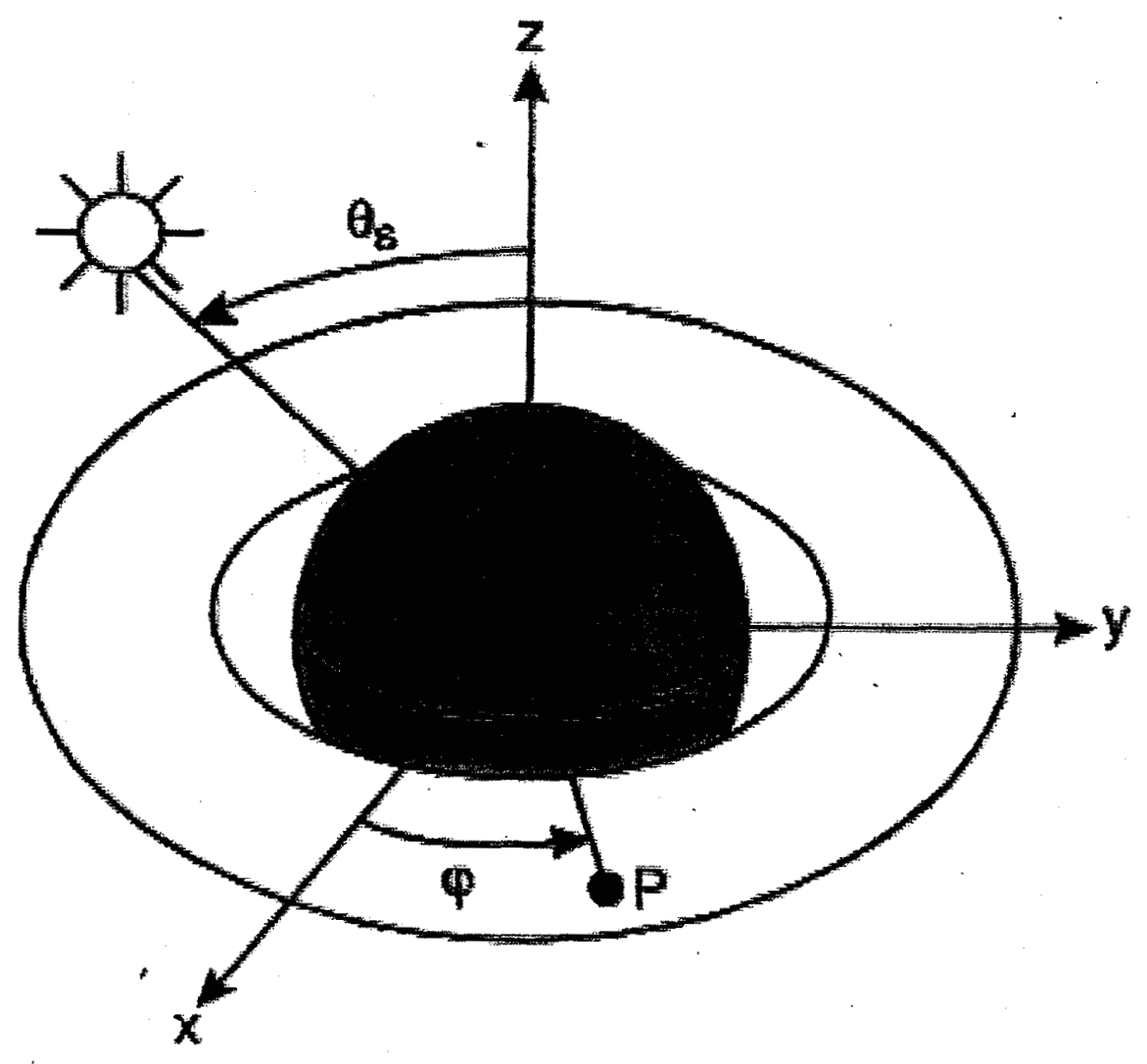

Figure 2. Coordinate system centered on Saturn. The $z$-axis points in the direction of the positive spin axis. The Sun lies in the $x$ - $z$ plane at a colatitude of $\theta_{s}$. A ring particle $P$ orbits in the equatorial plane at a distance $a$ from Saturn's center, and makes an angle $\phi=n t$ with the $x$-axis, where $n$ is the mean motion and $t$ is time.

The only term in $b(\phi)$ which will give a secular (i.e., long-term) evolution for the semimajor axis is the $j=1$ term, because it depends on $\cos \phi$. The Yarkovsky-Schach acceleration $\mathbf{f}_{Y S}$ of a spherical particle will then be given approximately by

$$
\mathbf{f}_{Y S}=-\left(1-A_{V}\right) B^{\prime} b_{1} F_{S u n} \cos (\phi-\delta)\left(\hat{\mathbf{r}}_{\mathbf{S}} \bullet \hat{\mathbf{s}}\right) \hat{\mathbf{s}}
$$


where $B^{\prime}$ and $\delta$ are an amplitude and a lag angle determined by the size and thermal properties of the particle and $A_{V}$ is its albedo in the visible. In effect the thermal inertia rotates the shadow by an angle $\delta$. The along-track acceleration $S_{Y S}$ is given by $S_{Y S}=\left(\mathbf{f}_{Y S} \bullet \hat{\mathbf{t}}\right)$, where $\hat{\mathbf{t}}=-\sin \phi \hat{\mathbf{x}}+\cos \phi \hat{\mathbf{y}}$ is the unit vector tangent to the orbit. Working out all the geometrical factors yields and averaging $\phi$ over one revolution about Saturn yields $\overline{S_{Y S}}$ being proportional to

$$
\begin{aligned}
& -2\left(\hat{\mathbf{r}}_{\mathbf{S}} \bullet \hat{\mathbf{s}}\right) \overline{(\hat{\mathbf{s}} \bullet \hat{\mathbf{t}}) \cos (\phi-\delta)} \\
& =+\sin \theta_{S} \sin ^{2} \theta_{p} \cos ^{2} \lambda_{p} \sin \delta+\cos \theta_{S} \sin \theta_{p} \cos \theta_{p} \cos \lambda_{p} \sin \delta \\
& -\sin \theta_{S} \sin ^{2} \theta_{p} \sin \lambda_{p} \cos \lambda_{p} \cos \delta-\cos \theta_{S} \sin \theta_{p} \cos \theta_{p} \sin \lambda_{p} \cos \delta
\end{aligned}
$$

For time scales longer than the orbital period, all but the first term on the right side of the above expression will drop out when averaging over longitude $\lambda_{p}$, because of precession. Note that the first term is greater than or equal to zero, so that $\bar{S}_{Y S} \geq 0$; the Yarkovsky-Schach effect tends to increase the semimajor axis $a$ of the particle. Thus the Yarkovsky-Schach effect pumps angular momentum into the rings. Averaging this term over all possible spin axis orientations gives the double average $\left\langle\bar{S}_{Y S}\right\rangle=+\left(1-A_{V}\right) F_{\text {Sun }}$ $B^{\prime} b_{1} \sin \theta_{S} \sin \delta / 3$. Lastly, because Saturn's equator is tilted by $26.73^{\circ}$ with respect to its orbital plane, $b_{1} \sin \theta_{s}$ must be averaged over one Saturnian year to account for the changing shadow geometry.

The diurnal Yarkovsky effect will average out to zero over all spin axis orientations. The averaged along-track acceleration due to the seasonal Yarkovsky effect is $\left\langle\bar{S}_{T D}\right\rangle=-\Xi$ $\left(1-A_{I R}\right) B^{\prime} F_{S u n} R_{S}^{2} \sin \delta / 6 a^{2}$ for circular, equatorial orbits (3), where $R_{S}=60,330 \mathrm{~km}$ is the radius of Saturn, $a$ is the semimajor axis of the particle, $A_{I R}$ is the albedo in the infrared, and the factor $\Xi=1.79 \pm 0.09$ is the amount that Saturn's thermal radiation exceeds the solar 
input (7). The subscript "TD" stands for "Thermal Drag," the original name for the seasonal Yarkovsky effect $(I)$.

For spherical particles $B^{\prime}$ is given by

$$
B^{\prime} \cong\left(\frac{4 \varepsilon_{l R} \sigma T_{0}^{3}}{9 c \rho K}\right) \sqrt{\frac{C_{1}^{2}+C_{2}^{2}}{C_{3}^{2}}}
$$

in the theory which relates temperature linearly to flux, where $c=3 \times 10^{8} \mathrm{~m} \mathrm{~s}^{-1}$ is the speed of light, $\sigma=5.67 \times 10^{-8} \mathrm{~W} \mathrm{~m}^{-2} \mathrm{~K}^{-4}$ is the Stefan-Boltzmann constant, $\rho$ is the particle's density, $K$ is its thermal conductivity, and $T_{0}$ is its average temperature, while $\varepsilon_{I R}$ is its infrared emissivity. Also, $\tan \delta=C_{2} / C_{1}$, where $C_{1}, C_{2}$, and $C_{3}$ are functions which appear in the spherical Bessel function formulation of the temperature and depend in a complicated way on the particle's radius $R$ and thermal properties (3).

The total along-track acceleration is $\langle\bar{S}\rangle=\left\langle\bar{S}_{Y S}\right\rangle+\left\langle\bar{S}_{T D}\right\rangle$. Figure 3 plots $\langle\bar{S}$ $>$ for a water ice "fluff ball" with the particular values $R=1 \mathrm{~cm}, T_{0}=90 \mathrm{~K}, \rho=380 \mathrm{~kg} \mathrm{~m}^{-3}$, $\varepsilon_{I R}=1, A_{V}=A_{I R}=0$, and $K=2.2 \times 10^{-4} \mathrm{~W} \mathrm{~m}^{-1} \mathrm{~K}^{-1}(8,9)$; but where the curve crosses the horizontal axis is independent of these parameters so long as $A_{V}=A_{I R}$, because the Yarkovsky forces vary in the same way for both effects. The curve crosses the axis in two places, at distances $a_{B C}$ and $a_{\mathrm{AO}}$. The region in between is where the net force on the particle is greater than zero and where rings would be expected. Beyond $a_{A O}$ the seasonal Yarkovsky effect predominates and a particle would be forced back inward into the ring system. At distances less than $a_{B C}$ the seasonal effect again predominates, and particles would fall onto Saturn. 


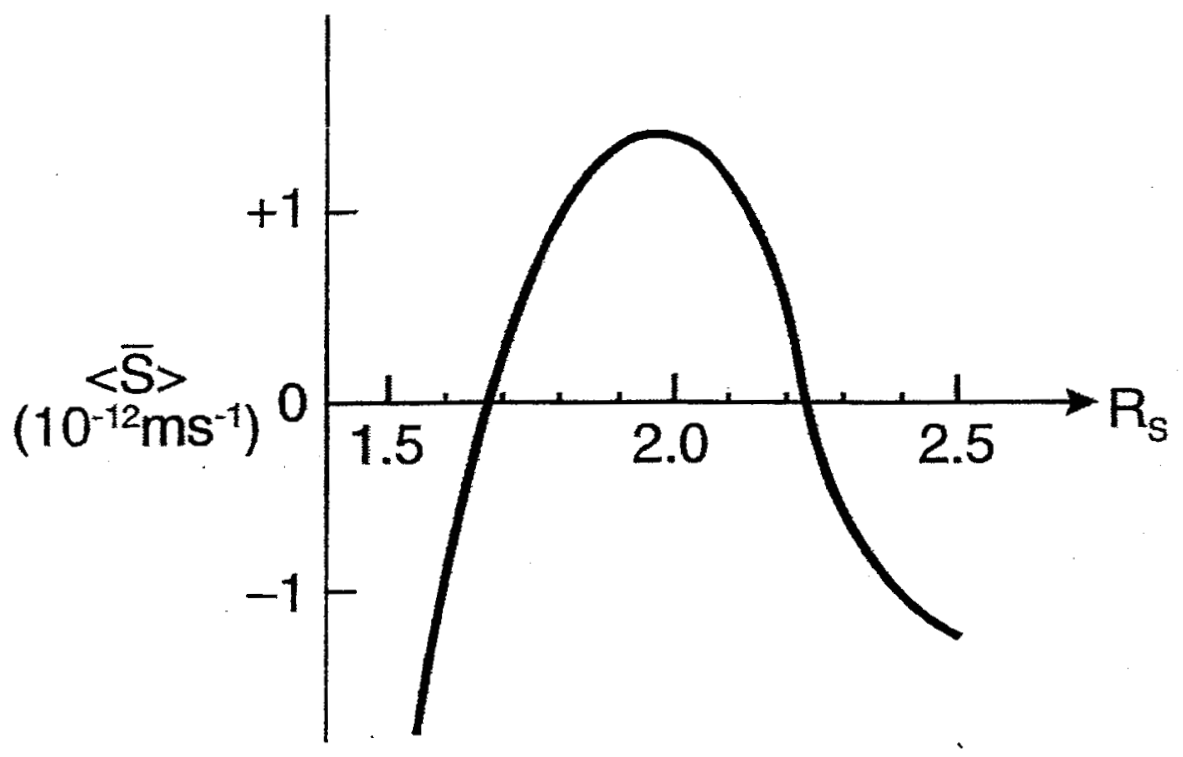

Figure 3. Averaged along-track acceleration $\langle\bar{S}\rangle$ as a function of $a$. Semimajor axis $a$ is measured in units of Saturn's radius $R_{s}$. The acceleration is the sum of the Yarkovsky-Schach effect and the seasonal Yarkovsky effect. For Saturn's current heat output $\langle\bar{S}\rangle$ is positive between $1.68 R_{S}$ and $2.23 R_{S}$. For values of $a>2.23 R_{S}$ ring particles would be forced back into the rings, while those with $a<1.68 R_{S}$ would fall on Saturn.

For the above parameters, the Yarkovsky forces predict the outer boundary to be at $a_{A O}=2.23 R_{S}$; the observed outer edge of the A ring is at $2.267 R_{S}$, where there is a 7:6 resonance, and the two values agree to with $2 \%$. The Yarkovsky inner boundary is at $a_{B C}=$ $1.68 R_{S}$; the boundary of the $\mathrm{B}$ and $\mathrm{C}$ rings is at $1.525 R_{S}(10,11)$, so that these two values agree to within $10 \%$. Apart from the resonance, the differences might be attributable to unequal visible and infrared albedos or to $\Xi$ being less than $1.79 ; \Xi=1.70$, for instance, yields $a_{A O}=2.34 R_{S}$ and $a_{B C}=1.54 R_{S}$. It is also of interest to note that the net acceleration reaches a maximum at $1.98 R_{S}$, which is close to the center of the Cassini gap at $1.987 R_{S}$.

The graph given by Figure 3 indicates that the outer edge $a_{A O}$ is the most stable position for the particles. So why haven't all the particles migrated there? The answer may 
be that the A and B rings are "full": collisions make the rings want to spread out into a near monolayer of particles which are as tightly packed as ring dynamics allows. The restoring forces at the outer edge of the A ring increase with distance until about $2.80 R_{S}$ (where they start leveling off), so that spreading much beyond the outer edge of the A ring is difficult. On the other hand, at the inner boundary $a_{B C}$ once particles cross the inner edge of the $\mathrm{B}$ ring into the $\mathrm{C}$ ring, the seasonal Yarkovsky effect drags them inward, and it is an easy trip to Saturn. Thus one can envision the following "reservoir" model for the A and B rings: they are filled with particles. Collisions cannibalize the larger particles and make smaller particles. The new particles elbow the older ones for room. The older ones find it easiest to spiral into the planet, rather than extend the A ring outward.

This may also explain why the $C$ ring, which is located where thermal drag predominates, is more sparsely populated than the $\mathrm{A}$ or $\mathrm{B}$ rings: the particles in the $\mathrm{C}$ ring are transients, on their way to Saturn. If this is so, then there might be a continuous rain of icy meteoroids all around the planet's equator, whose luminous fireballs would give the impression of a "Ring of Fire." The Ring of Fire might be visible to Cassini's cameras when imaging the equator on the night side.

Figure 4 graphs semimajor axis $a$ vs. time $t$ for particles with radii $R=0.1 \mathrm{~cm}, 1 \mathrm{~cm}$, and $10 \mathrm{~cm}$ using the equation

$$
\frac{d a}{d t}=\frac{2\langle\bar{S}\rangle}{n}
$$

(12). The particles start their journey at $1.70 R_{S}$. It is assumed that the only forces operating on the particles are Saturn's gravity and the Yarkovsky forces. The orbit evolves the quickest for the $1 \mathrm{~cm}$ particle, which has nearly ideal properties for the operation of the Yarkovsky effects. The rings consist predominantly of particles in the size range $1-10 \mathrm{~cm}$, close to the ideal. 


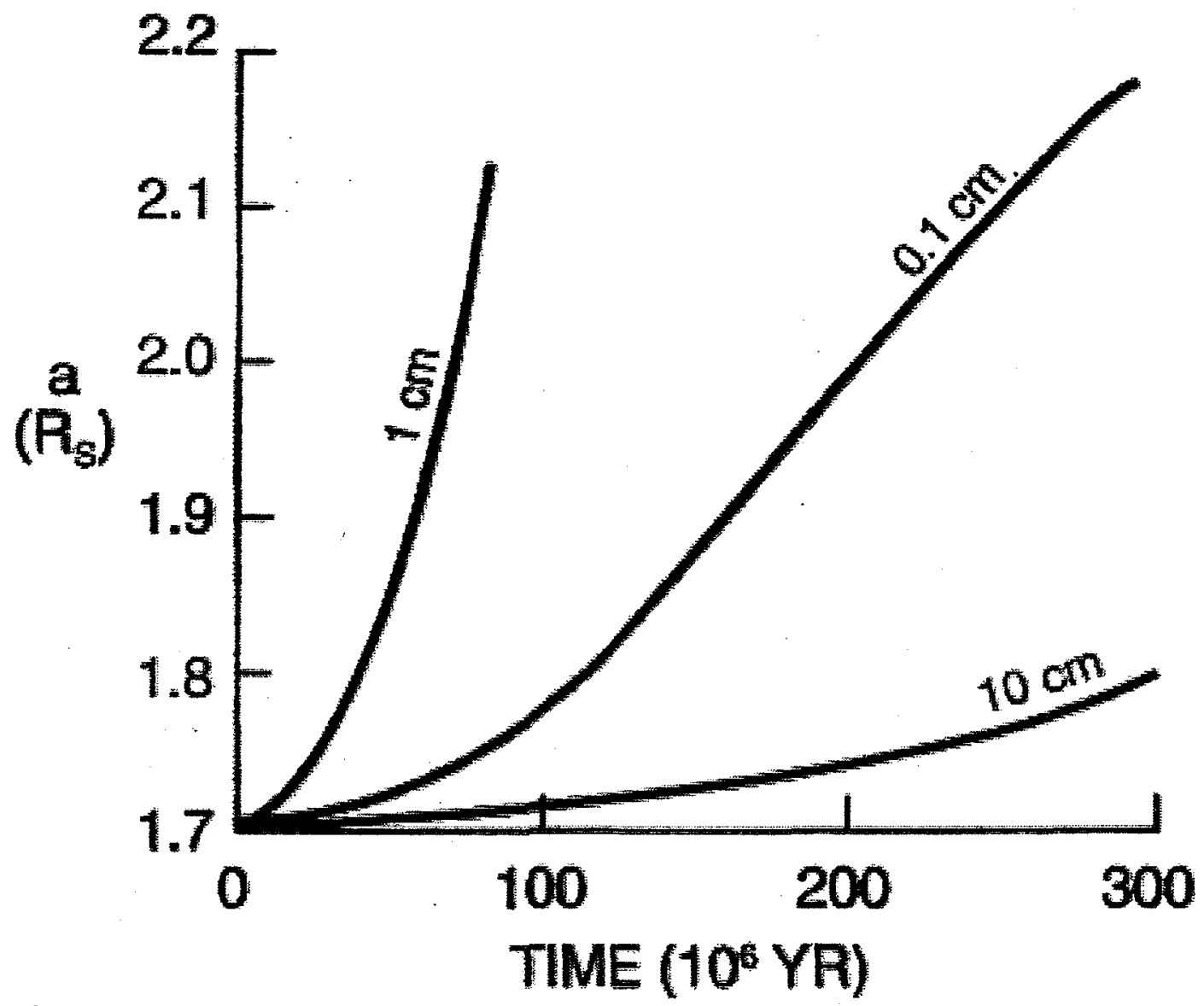

Figure 4. Semimajor axis $a$ as a function of time $t$ for spherical particles with radii $0.1 \mathrm{~cm}, 1 \mathrm{~cm}$, and $10 \mathrm{~cm}$, assuming the particles are "fluff balls" and act under Yarkovsky forces and Saturn's gravity only. No collisions or gravity from the moons are assumed. The particles are initially at $1.7 R_{S}$.

Collisions will slow down the rate of Yarkovsky evolution by degrading the coherence between thermal forces and the orbit. However, because both the YarkovskySchach effect and the seasonal Yarkovsky effect have the same functional dependence on $\phi$, they will decohere the orbit-spin coupling in lockstep, so that the positions of $a_{B C}$ and $a_{A O}$ in Figure 3 will remain unchanged.

Estimates of how often collisions take place range from 1 to 1000 times per orbit (13-15). Obviously bumping is not serious for sparse, and probably not to serious for close-packed particles. To see this for the close-packing, assume the particles collide 1000 times per orbit. This is about once every 30 seconds. The particles most likely rotate with 
angular speeds close to synchronous rotation (16-19). If each collision causes the spin axis to precess in a new direction at the same rate as rotation, the axis will rotate only through $0.4^{\circ}$ before being struck again. With Brownian motion, the angular position will grow like $N^{1 / 2}$, where $N$ is the number of collisions. So after one orbit and 1000 collisions, the axis will have moved through only $12^{\circ}$, which is not terribly significant for the direction of Yarkovsky forces.

So probably somewhere in between sparse and close-packed is the regime where coherence degrades the most. This leads to the idea of Yarkovsky-collision feedback: when the particles huddle together and collisions become frequent, Yarkovsky efficiency improves and spreads the particles out. Hence Yarkovsky may help explain the spacing between ring particles. Collisions, and the gravitational perturbations from Saturn's many moons, await future research.

Yarkovsky may constrain the age of Saturn's rings. At present $\Xi=1.79 \pm 0.09$; but in the past $\Xi$ was larger, due to the higher heat flow inside Saturn (20), so that the seasonal Yarkovsky effect was greater and the zone where $<\bar{S}>$ is positive was smaller; $a_{B C}$ and $a_{A O}$

were closer together. At $\Xi \cong 1.90, a_{B C}=a_{A O}$ and the rings are unstable; the seasonal Yarkovsky effect predominates at all distances, and ring particles must fall on Saturn. Thus the Yarkovsky effects imply the rings are young, perhaps on the order of $\sim 10^{8}$ years old, in agreement with other mechanisms (15). Possibly they were created in the cometary shower which occurred $36 \times 10^{6}$ years ago $(21,22)$.

\section{References and Notes}

1. D. P. Rubincam, J. Geophys. Res. 92, 1287 (1987).

2. D. P. Rubincam, J. Geophys. Res., 100, 1585 (1995).

3. D. P. Rubincam, J. Geophys. Res. 103, 1725 (1998).

4. W. F. Bottke, D. Vokrouhlicky, D. P. Rubincam, M. Broz, in Asteroids III, W. F. Bottke, A. Cellino, P. Paolicchi, R. P. Binzel, Eds. (Univ. of Arizona Press, Tucson 2002), pp. 395-408. 
5. D. P. Rubincam, Celest. Mech. 26, 361 (1982).

6. D. P. Rubincam, in Relativistic Gravitational Experiments in Space, M. Demianski, C. W. F. Everitt, Eds. (World Scientific, River Edge, NJ, 1993), pp. 195-209.

7. B. J. Conrath, R. A..Hanel, R. E. Samuelson, in Origin and Evolution of Planetary and Satellite atmospheres, S. K. Atreya, J. B. Pollack, M. S. Matthews, Eds. (Univ. of Arizona Press, Tucson, 1989), pp. 513-538.

8. P. A. Rosen, G. L. Tyler, E. A. Marouf, J. J. Lissauer, Icarus 93, 25 (1991).

9. L. Spilker, C. Ferrari, J. N. Cuzzi, M. Showalter, J. Pearl, B. Wallis, Planet. Space Sci. 51, 929 (2003).

10. J. N. Cuzzi, J. J. Lissauer, L. W. Esposito, J. B. Holberg, E. A. Marouf, G. L. Tyler, A. Boischot, in Planetary Rings, R. Greenberg, A. Brahic, Eds. (Univ. of Arizona Press, Tucson, 1984), pp. 73-199.

11. L. W. Esposito, J. N. Cuzzi, J. B. Holberg, E.A. Marouf, G. L. Tyler, C. C. Porco, in Saturn, T. Gehrels, M. S. Matthews, Eds. (Univ. of Arizona Press, Tucson, 1984), pp. 463-545.

12. V. M. Blanco, S. W. McCuskey, Basic Physics of the Solar System (Addison-Wesley, Reading, MA, 1961).

13. J. Wisdom, S. Tremaine, Astron. J. 95, 925 (1988).

14. H. Salo, Icarus 96, 85 (1992).

15. L. Dones, in Encyclopedia of Planetary Sciences, I. H. Shirley, R. W. Fairbridge, Eds. (Chapman \& Hall, London, 1997), pp. 719-723.

16. G. Shukhman, Sov. Astron. 28, 574 (1984).

17. D. Richardson, Mon. Not. R. Astron. Soc. 269, 493 (1994).

18. H. Salo, Icarus 117, 287 (1995).

19. S. Araki, Icarus 90, 139 (1991).

20. J. B. Pollack, G. Consolmagno, in Saturn, T. Gehrels, M. S. Matthews, Eds. (Univ. of Arizona Press, Tucson, 1984), pp. 811-866.

21. K. A. Farley, A. Montanari, E. M. Shoemaker, C. S. Shoemaker, Science 280, 1250 (1998).

22. I thank William F. Bottke and Luke Dones for valuable information, and Susan Fricke and Susan Poulose for excellent programming support. 